\title{
A Electrocatálise: um domínio científico-tecnológico
}

\section{Introdução}

À Electrocatálise tem sido dado, na última década, lugar de relevo em muitos congressos e reunið̌es científicas, tendo mesmo sido dedicadas exclusivamente ao tema duas grandes conferências [1,2]. Alguns artigos de revisão têm sido publicados [3-5], mas é sobretudo o número de publicaçð̃es, resultantes de projectos em curso nesta área, que justificam o interesse e a actualidade do tema.

\section{O que é a Electro(catálise)?}

Para os processos electroquímicos tal como para os processos químicos, catalisar significa aumentar a velocidade do processo sem consumir "mais energia", ou mesmo reduzindo o valor da energia consumida.

Para os processos químicos a velocidade é uma função exponencial da energia livre de activação segundo a equação de Arrhenius,

$v_{c}=c \frac{k_{B} T}{h} e^{-\Delta^{*} G^{\circ} / R T}$

em que $\Delta^{*} G^{0}$ representa a energia livre de activação, $\mathrm{T}$ a temperatura absoluta, $\mathrm{k}_{\mathrm{B}}$ a constante de Boltzman, $\mathrm{h}$ a constante de Planck e c a concentração da espécie reactiva.

Porém, para os processos electroquímicos a velocidade, além de ser função da energia livre de activação é também, função do potencial, pois que:

$\mathrm{v}_{\mathrm{e}}=\frac{\mathrm{i}}{\mathrm{nF}}\left(\frac{\mathrm{k}_{\mathrm{B}} \mathrm{T}}{\mathrm{h}} \mathrm{e}^{-\Delta^{*} \mathrm{G}^{\circ} / \mathrm{RT}}\right) \mathrm{e}^{-\alpha \mathrm{nF} \Delta \Phi / \mathrm{RT}}$

em que $\alpha$ representa o coeficinete de transferência de carga $(\alpha<1)$; i a densidade de corrente devida ao processo de transferência de carga e $\Delta \Phi$ a diferença de potencial na interface eléctrodo/solução. Assim, a catálise de um processo electroquímico pode ser conseguida, quer através de uma diminuição de $\Delta^{*} \mathrm{G}^{\circ}$, quer através de uma redução do sobrepotencial $\eta\left(\eta=\Delta \Phi-\Delta \Phi_{\mathrm{e}}\right)$, ou ainda, através de ambos. Como para um processo electroquímico se tem, de acordo com Tafel,

$|\eta|=\frac{2,303 \mathrm{RT}}{\alpha \mathrm{nF}} \log \left(\mathrm{i} / \mathrm{i}_{\mathrm{o}}\right)$

então, um bom catalisador é o que conduz a um valor de $\mathrm{i}_{\mathrm{o}}$ elevado e/ou a um valor de $\alpha$ pequeno.

Em Electrocatálise, a actividade de dois catalisadores, para um determinado processo, deve ser comparada para o mesmo valor de sobrepotencial, que pode ser o sobrepotencial $\eta=0\left(\Delta \Phi=\Delta \Phi_{\mathrm{e}}\right)$, o que significa comparar valores das densidades das correntes de troca, $\mathrm{i}_{\mathrm{o}}$.
A Electrocatálise por tratar, em geral, dos processos que ocorrem na interface eléctrodo/solução, processos heterogéneos de transferência de carga, apresenta certas analogias com a catálise enzimática e também com a catálise heterogénea sólido/gás.

Há, contudo, diferenças importantes entre a Electrocatálise e a catálise química heterogénea. Assim, em Electrocatálise trabalha-se com temperaturas relativamente baixas $\left(\mathrm{t}<150^{\circ} \mathrm{C}\right)$, em comparação com temperaturas utilizadas em catálise química $\left(150-500^{\circ} \mathrm{C}\right)$. Também as energias de activação associadas aos processos de Electrocatálise são muito menores do que as associadas aos processos de catálise química $\left(5-35 \mathrm{kcal} \mathrm{mol}^{-1} \mathrm{em}\right.$ comparação com os valores de $10-100 \mathrm{kcal} \mathrm{mol}^{-1}$ ).

Em Electrocatálise o calor de activação $\Delta^{*} \mathrm{H}$ para um dado processo pode ser diminuído, usando para o efeito o sobrepotencial $\eta$, pois que,

$\Delta^{*} \mathrm{H}_{\eta}=\Delta^{*} \mathrm{H}_{\mathrm{o}}-\alpha \mathrm{nF}$

sendo $\Delta^{*} \mathrm{H}_{\mathrm{o}}$ o calor de activação na ausência de sobrepotencial aplicado ao sistema.

O termo Electrocatálise é, em geral, aplicado no seu significado mais restrito, ou seja, processos de eléctrodo que envolvem ligaçð̃es ou pelo menos interacçð̃es fortes dos reagentes, produtos ou intermediários com a superfície do eléctrodo. O catalisador é assim, o próprio eléctrodo ou o eléctrodo modificado.

\section{As Origens da Electrocatálise}

A Electrocatálise nasceu para dar resposta a um certo número de problemas de índole tecnológica, em particular, problemas associados ao desenvolvimento das células de combustível, à produção do $\mathrm{O}_{2} \mathrm{e}$ do $\mathrm{H}_{2}$ por electrólise da água, à extracção do $\mathrm{Al}$ e à produção do $\mathrm{Cl}_{2}$, de entre outros.

Em qualquer processo de electrólise a energia consumida é proporcional à diferença do potencial $\mathrm{V}$, apresentada pelos terminais da célula electrolítica e relacionado com os sobrepotenciais dos processos anódico e catódico pela igualdade,

$\mathrm{V}=\Delta \mathrm{E}_{\mathrm{e}}-\eta_{\mathrm{a}}-\eta_{\mathrm{c}}-\mathrm{IR}$

em que, $\Delta \mathrm{E}_{\mathrm{e}}$ representa a diferença entre os potenciais de equilíbrio dos dois processos, $\eta_{\mathrm{a}}$ e $\eta_{\mathrm{c}}$ os sobrepotenciais associados respectivamente aos processos anódico e catódico e IR a queda óhmica.

Tornar a célula mais eficiente implica reduzir $\eta_{\mathrm{a}}, \eta_{\mathrm{c}}$ bem como IR.

\footnotetext{
a Departamento de Química da Faculdade de Ciências, Universidade de Lisboa
} 
Para as células de combustivel os alcoois (sob-produtos da biomassa) são combustíveis anódicos de grande interesse, assim como o são os hidrocarbonetos. Como combustíveis catódicos tem-se o $\mathrm{O}_{2}$ que pode ser reduzido e o ião $\mathrm{H}^{+}$, que por redução dá $\mathrm{H}_{2}$.

Cada um dos processos de eléctrodo mencionado, justifica per si um estudo exaustivo, com o objectivo de para eles serem encontrados bons catalisadores. Contudo, mesmo quando um bom catalisador já foi encontrado para um dos processos, o funcionamento da célula de combustível pode ainda ser pouco eficiente, em particular, quando para o mesmo valor de sobrepotencial os dois processos têm velocidades muito diferentes. Este é um dos problemas que está associado à baixa eficiência das células de $\mathrm{O}_{2} / \mathrm{H}_{2}$ em meio ácido, com eléctrodos de platina e a temperaturas não muito elevadas. A velocidade é limitada pela velocidade específica da reaç̧ão de redução do $\mathrm{O}_{2}$. Assim, para o mesmo sobrepotencial há uma grande diferença na velocidade dos dois processos, a oxidação do $\mathrm{H}_{2}$ é muito mais rápida que a redução do $\mathrm{O}_{2}$, pelo que há perda de energia no processo global.

A oxidação do $\mathrm{HCOOH}$ e do $\mathrm{CH}_{3} \mathrm{OH}$ tem sido também objecto de numerosos estudos, no domínio da Electrocatálise. Compreender o mecanismo da oxidação catalítica do $\mathrm{HCOOH}$ e do $\mathrm{CH}_{3} \mathrm{OH}$ é fundamental para a concepção de novos catalisadores mais económicos e estáveis.

\section{Objectivos da Electrocatálise}

A Electrocatálise pretende encontrar respostas para questðes como:

- O que determina a actividade catalítica de um catalisador?

\section{- Como controlar essa actividade?}

Quando a estas grandes questðes for dada uma resposta em termos das propriedades físico-químicas e estruturais dos materiais, então, será possível conceber e produzir, de um modo racional, bons catalisadores. Acontece, porém, que as questðes que se colocam são talvez demasiado ambiciosas e o interesse tecnológico dos processos que motivaram o nascimento da Electrocatálise não pode esperar o tempo necessário para que as teorias sejam formuladas e testadas. Assim, a concepção de novos catalisadores é ainda feita, senão de um modo empírico, pelo menos, numa base semi empírica, ou seja, experimentam-se para os diferentes processos materiais que verifiquem alguns dos requisitos que a experiência tem demonstrado serem decisivos para a actividade catalítica.

Acresce ainda, que um bom catalisador de um processo não o é, necessariamente de outro - a actividade catalítica tem que ver com o mecanismo da reacção a Electrocatálise apresenta especificidade.

\section{Que respostas têm sido encontradas?}

Os estudos de Electrocatálise têm concluído que os processos susceptíveis de serem catalisados de um modo mais significativo são os processos heterogéneos que envolvam a formação de ligaçðes ou interacçðes fortes entre o eléctrodo e os reagentes ou as espécies intermediárias. É o que se verifica na oxidação do $\mathrm{HCOOH}$, cujo processo é descrito, segundo Capon e Parsons [6], pelas equaçð̃es:

$$
\begin{aligned}
& \mathrm{HCOOH} \underset{\text { lento }}{\longrightarrow} \mathrm{Pt}-\mathrm{H}+\mathrm{Pt}-\mathrm{COOH} \\
& \mathrm{Pt}-\mathrm{H} \underset{\text { rápido }}{\longrightarrow} \mathrm{H}^{+}+\mathrm{e} \\
& \mathrm{Pt}-\mathrm{COOH} \\
& \text { (a) } \\
& \text { (b) } \\
& \underset{\mathrm{Pt}}{\mathrm{Pt}} \underset{\mathrm{Pl} \text { (PHO }}{\mathrm{CHO}}
\end{aligned}
$$

A etapa (6), que controla a cinética do processo, é um processo dissociativo que envolve ligaçð̄es ao eléctrodo de Pt. Também a espécie ( $\mathrm{Pl})$ envolve a formação de ligaçð̃es ao eléctrodo, só que esta é uma espécie não desejável para o bom rendimento do processo.

Para os processos referidos e em particular, para a oxidação do $\mathrm{HCOOH}$ tem-se verificado que:

Os metais de transição e, em especial, os metais nobres são os melhores catalisadores variando contudo, a actividade de metal para metal, como o demonstram os resultados da Tabela 1

TABELA 1

Densidade das correntes de pico para a oxidaçâo do $\mathrm{HCOOH}$ nos metais nobres [6]

\begin{tabular}{cc}
\hline Metal & $2 \mathrm{ip} / \mathrm{mA} \mathrm{cm}^{-2}$ \\
\hline $\mathrm{Pt}$ & 140 \\
$\mathrm{Pd}$ & 6.4 \\
$\mathrm{Rh}$ & 24.6 \\
$\mathrm{Ir}$ & 0.8 \\
$\mathrm{Au}$ & 0.1 \\
\hline
\end{tabular}

As diferenças de actividade encontradas para os metais nobres (Tabela 1) têm sido atribuídas ao $n .^{\circ}$ de electrōes d não emparelhados e às diferenças dos níveis energéticos dos referidos electrőes, factores esses que por sua vez se reflectem nos valores do calor de sublimação do metal e no valor do calor de formação das espécies adsorvidas. As curvas como as da Figura 1 designadas por curvas "vulcano", mostram também que a Pt é o metal nobre com maior actividade catalitica para a oxidação do $\mathrm{HCOOH}$, como aliás o é para um grande número de processos susceptíveis de serem catalisados.

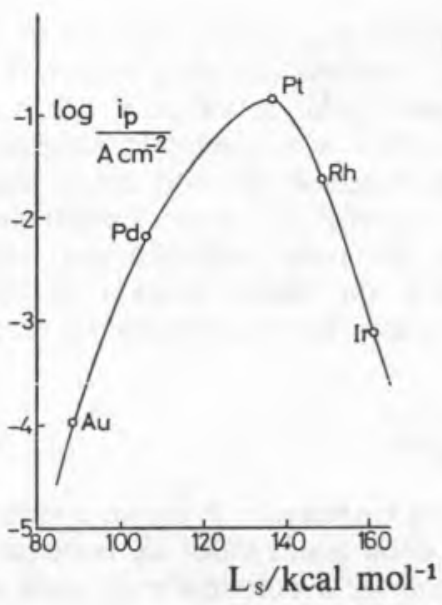

FIGURA 1

Densidade da corrente de pico para a oxidação do $\mathrm{HCOOH}(0.265 \mathrm{M})$ em função do calor latente de sublimação do metal [7] 
A actividade catalítica depende da estrutura cristalográfica do metal

Neste domínio os monocristais são uma ferramenta preciosa para demonstrar os efeitos da estrutura. Clavilier e col. [8] forma pioneiros nestes estudos. Os resultados apresentados na Figura 2 são bem ilustrativos das diferenças devidas à orientação estrutural da superfície do eléctrodo.
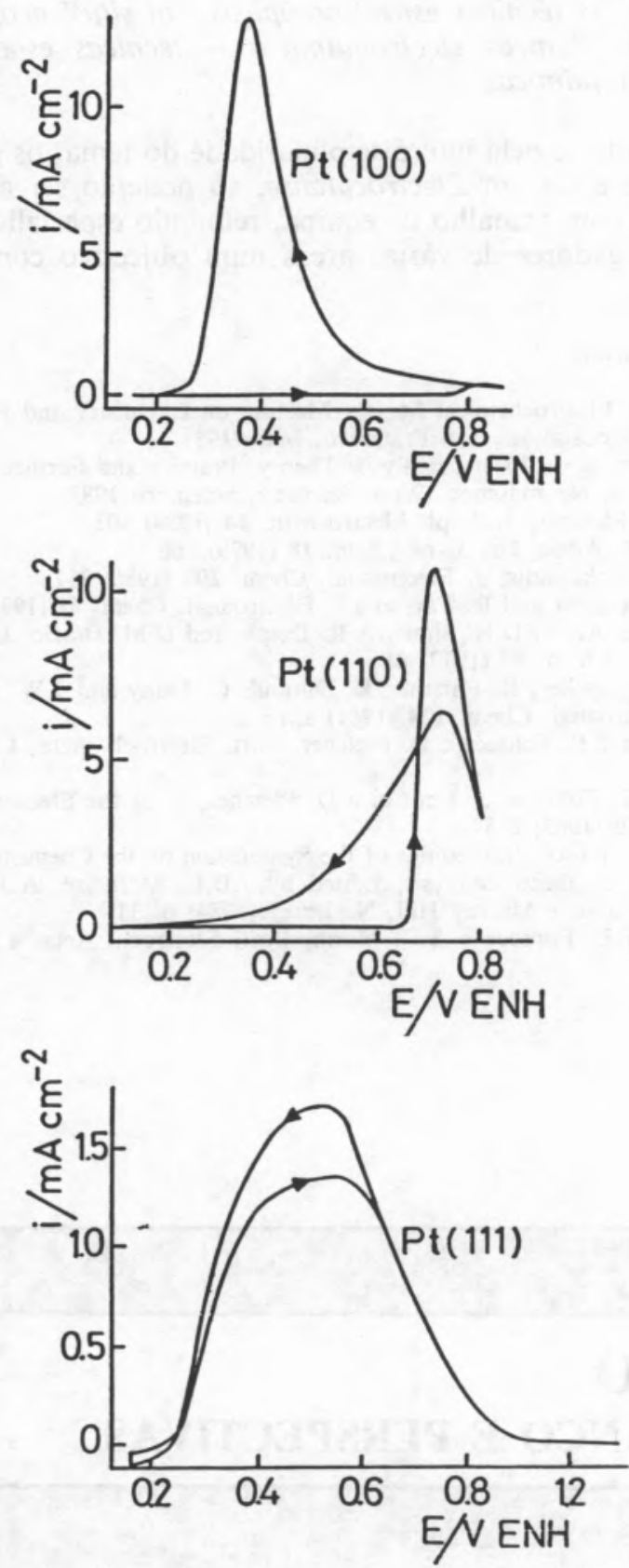

FIGURA 2

Voltamogramas cíclicos da oxidação

do $\mathrm{HCOOH}\left(0.1 \mathrm{~mol} \mathrm{dm}^{-3}\right) \mathrm{em} \mathrm{Pt} / 0.5 \mathrm{~mol} \mathrm{dm}^{-3} \mathrm{H}_{2} \mathrm{SO}_{4}$, nas faces cristalinas indicadas (Resultados de Clavilier et al. [8])

A deposição de monocamadas ou sub-monocamadas de adatomos de metais pesados modificam a actividade catalítica dos metais nobres - electrocatálise por adatomos

$\mathrm{O}$ efeito dos adatomos tem sido estudado por diferentes escolas. A Figura 3 exemplifica o referido efeito.

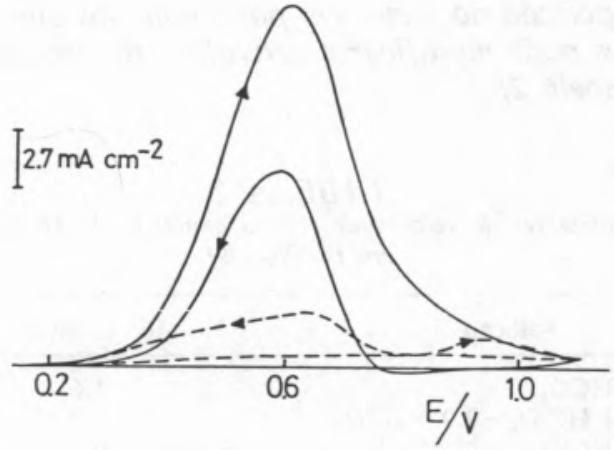

FIGURA 3

Voltamogramas cíclicos da oxidação

do HCOOC (0.1M) em Pt, na ausência (--) e na presença (-) de $\mathrm{Pb}, v=0.1 \mathrm{~V} \mathrm{~s}^{-1}[9]$

Observa-se assim, que a presença do $\mathrm{Pb}$ na platina faz aumentar a densidade de corrente de pico para a oxidação do $\mathrm{HCOOH}$ de um factor de cerca de 30 .

Contudo, verifica-se também que a actividade induzida depende do adatomo escolhido, como o demonstram as curvas da Figura 4.

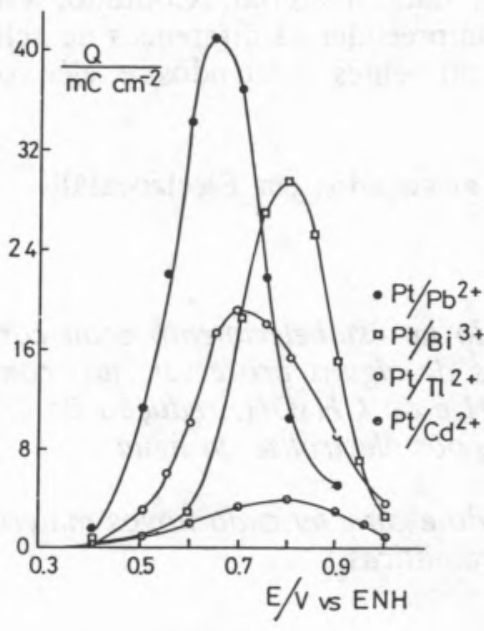

FIGURA 4

Curvas da carga (ls) para a oxidação do $\mathrm{HCOOH}$ em função do potencial para vários metais - adatomos - ([10]

A actividade induzida pelos adatomos é função do grau de recobrimento do eléctrido (vejam-se os resultados da Figura 5)

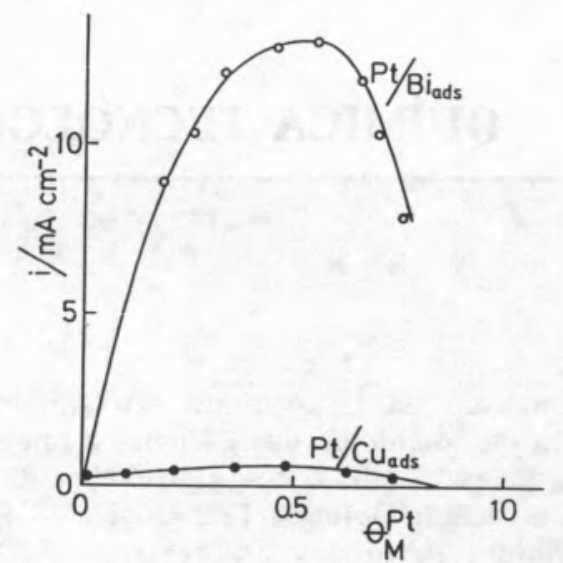

FIGURA 5

Densidade de corrente para a oxidação do $\mathrm{HCOOH} 1 \mathrm{M} \mathrm{em} \mathrm{H}_{2} \mathrm{SO}_{4} 0.5 \mathrm{M}$, para $E=0.25 \mathrm{~V}$ e $t=40^{\circ} \mathrm{C}$ em função do grau de recobrimento da Pt por adatmos (Resultados de Motoo et al [11]) 
A composição do meio, em particular, no que se refere $a$ aniões pode modificar a actividade de um catalisador (ver Tabela 2)

$T A B E L A 2$

Constantes de velocidade para a oxidação do $\mathrm{HCOOH}$ em $\mathrm{Pt} / \mathrm{Pb}$ ads $[9]$

\begin{tabular}{cc}
\hline Solução & $10^{2} \mathrm{k} / \mathrm{cm} \mathrm{s}^{-1}$ \\
\hline $1 \mathrm{M} \mathrm{HClO}_{4}$ & 1.6 \\
$0.1 \mathrm{M} \mathrm{HClO}_{4}+0.9 \mathrm{NaClO}_{4}$ & 3.2 \\
$1 \mathrm{M} \mathrm{HClO}_{4}+10^{-5} \mathrm{M} \mathrm{Cl}^{-}$ & 0.7 \\
\hline
\end{tabular}

Verifica-se, da análise da Tabela 2, quue o ião $\mathrm{Cl}^{-}$diminui significativamente a velocidade de oxidação do $\mathrm{HCOOH}$. O efeito foi atribuído à adsorção do ião $\mathrm{Cl}^{-}$ pela Pt $[9,12]$.

De um modo geral, pode dizer-se que os estudos exaustivos que vêm sendo realizados no domínio da Electrocatálise, têm permitido chegar a algumas conclusð̃es sobre os factores que determinam a actividade catalitica de um dado material. Contudo, estamos ainda longe de compreender as diferenças de actividade reveladas por diferentes eléctrodos e eléctrodos modificados.

\section{Objectivos alcançados em Electrocatálise}

Tem-se:

- Avançado no estabelecimento elou confirmação de mecanismos de alguns processos, tais como, oxidação do $\mathrm{HCOOH}$ e do $\mathrm{CH}_{3} \mathrm{OH}$, redução do $\mathrm{O}_{2}$ e produção de $\mathrm{O}_{2}$ e $\mathrm{H}_{2}$ por electrólise da água

- Produzido e caracterizado novos materiais com propriedades catalíticas
- Progredido na "performance" e eficiência das células de combustível, bem como nos processos de electrólise da água

Os objectivos alcançados em Electrocatálise, como em tantas outras áreas, dependem da teoria de que se dispð̃e e das técnicas capazes de dar informação. Neste sentido, surgem como meios promissores:

- (i) Os monocristais de diferentes materiais

- (ii) As técnicas espectroscópicas "in situ” acopoladas às técnicas electroquímicas - técnicas espectro-electroquímicas

Contudo, e pela interdisciplinaridade do tema, os grandes sucessos em Electrocatálise, só poderão ser alcançados com trabalho de equipa, reunindo especialistas e investigadores de várias áreas num objectivo comum.

\section{Referências}

[1) The Electrochemical Society Meeting on Chemistry and Physics of Electrocatalysis, San Francisco, Maio 1983

[2] Meeting on Electrocatalysis: Theory, Practice and Further Developments, Neunkischen, West Germany, Setembro 1983

[3] D. Pletcher, J. Appl. Electrochem. 14 (1984) 403

[4] R.R. Adzic, Isr. J. of Chem. 18 (1979) 166

[5] G. Kokkinidis, J. Electroanal. Chem. 201 (1986) 217

[6] A. Capon and R. Parsons, J. Electroanal. Chem. 45 (1973) 205 [7] R.R. Adzic, D.N. Simic, A.R. Despic and D.M. Drazic, J. Electroanal. Chem. 80 (1977) 81

[8] L. Clavilier, R. Parsons, R. Durand, C. Lamy and J.W. Leger, J. Electroanal. Chem. 124 (1981) 321

[9] Inês T.E. Fonseca e D. Pletcher, Port. Electroch. Acta, 1 (1983) 219

[10] Inês Fonseca, J. Lin-Cai e D. Pletcher, J. of the Electrochem. Soc. 130 (1983) 2187

[11] S. Motoo, Proceedings of the Symposium on the Chemistry and Physics of Electrocatalysis, Edited by J.D.E. McIntyre, A.T. Bell Laboratories e Murray Hill, N. Jersey (1984) p. 331

[12] I.T.E. Fonseca e A.C. Marin, Port. Electroch. Acta, 4 (1986) 131

\section{COLÓQUIO}

QUíMICA TECNOLÓGICA - BALANÇO E PERSPECTIVAS

Os Finalistas da Licenciatura em Química Tecnologica da Faculdade de Ciências de Lisboa, no âmbito da cadeira de Sociologia das Ciências e integrado nas actividades de fim de curso, realizam no próximo dia 4 de Maio, pelas 15 horas, no Salão Nobre da Reitoria da Universidade Clássica de Lisboa, um Colóquio subordinado ao tema: Química Tecnologica - Balanço e Perspectivas. Pretendem com esta iniciativa, divulgar esta licenciatura junto das Empresas do Sector Químico ou com ele relacionado, Instituições Universitárias e Público em Geral, avaliar das necessidades e especificidades das Empresas e Institutos de Investigação em termos da formação de recursos humanos na śrea da Química Tecnológica. 